\title{
Fracture Resistance of Immature Incisors Following Root Filling with Various Bioactive Endodontic Cements Using an Experimental Bovine Tooth Model
}

\author{
Pegah Sarraf1," $\quad$ Mohammad Hossein Nekoofar ${ }^{1,2} \quad$ Mohammad Saeed Sheykhrezae ${ }^{1, *} \quad$ Paul M. H. Dummer \\ ${ }^{1}$ Department of Endodontics, School of Dentistry, Tehran University \\ of Medical Sciences, Tehran, Iran, \\ 2School of Dentistry, College of Biomedical and Life Sciences, \\ Cardiff University, Cardiff, United Kingdom \\ Address for correspondence Mohammad Hossein Nekoofar, DDs, \\ MSc, DolBoE, PhD, School of Dentistry, Cardiff University, Cardiff \\ CF10 3XQ, UK (e-mail: nekoofarmh@cardiff.ac.uk).
}

Eur J Dent 2019;13:156-160

\begin{abstract}
Keywords

- fracture resistance

- mineral trioxide aggregate

- CEM Cement

- Biodentine
\end{abstract}

Objective The aim of this study was to compare the fracture resistance of immature bovine roots when using ProRoot MTA, CEM Cement, and Biodentine as root filling materials.

Materials and Methods An immature bovine tooth model was developed by removing the coronal and apical portions of 70 bovine incisors $8 \mathrm{~mm}$ above and $12 \mathrm{~mm}$ below the cementoenamel junction (CEJ). The specimens were then divided into five groups: ProRoot MTA, CEM Cement, Biodentine, gutta-percha/AH26 sealer, and control. All groups received a $5-\mathrm{mm}$ apical plug with a temporary restorative material. Then, the remaining root canal space was filled with one of the afore-mentioned materials. After setting, the specimens were mounted in acrylic resin. Then, $3 \mathrm{~mm}$ coronal to the $\mathrm{CE}$ from the buccal side of the teeth and at a $135^{\circ}$ angle to the long axis, the specimens were loaded until fracture.

Results The specimens in the Biodentine (2196 N) and ProRoot MTA (2103 N) groups had significantly greater fracture resistance in comparison to the control group ( $p=$ 0.01). No significant difference was found between CEM Cement, gutta-percha and sealer $\mathrm{AH} 26$, and control groups. No significant differences occurred between the four experimental groups $(p=0.45)$.

Conclusion Filling the root canal space with ProRoot MTA and Biodentine contributed to higher fracture resistance values.

\section{Introduction}

Immature teeth with pulp necrosis are susceptible to fracture due to their thin dentine walls and underdeveloped roots. ${ }^{1}$ Root canal treatment of such teeth with open apices and wide divergent apical walls is challenging. Conventionally, endodontic management of immature teeth has been performed using long-term intracanal application of calcium hydroxide. ${ }^{2}$ However, it has been reported that following long-term use of calcium hydroxide, a 50\% reduction in tooth

$\overline{{ }^{*} \text { Co-first }}$ authors. strength occurred over 1 year. ${ }^{3}$ In addition, as a consequence of the changes to the organic matrix in dentine, cervical root fracture may also occur. ${ }^{4,5}$ As an alternative treatment, calcium silicate-based cements such as mineral trioxide aggregate (MTA) have been suggested as apical barriers for onestep management of immature teeth with necrotic pulps. ${ }^{6-8}$ Following the placement of the apical barrier, the remainder of the canal is most often filled with gutta-percha and sealer. However, to improve the sealing of the middle and coronal portions of underdeveloped roots, it has been suggested that the remaining root canal system should be filled with a calcium silicate-based cement. ${ }^{9,10}$ In fact, it has been reported that 
this technique can reinforce the immature root structure ${ }^{11}$ because the cervical and middle portions of the root provide the bulk of the fracture resistance. Furthermore, since the thickness of the canal walls in the apical third is limited, root-end filling materials do not support direct occlusal loads. ${ }^{12}$ Biodentine (Septodont, St Maur-des-Fossés, France), a bioactive cement, was introduced as a dentine restorative material and pulp capping agent with the aim of improving several drawbacks associated with other calcium silicate cements, such as long setting time ${ }^{13}$ and difficult handling properties. ${ }^{14}$ Calcium-enriched mixture CEM Cement (BioniqueDent, Tehran, Iran) has also been developed to overcome the disadvantages of other calcium silicate materials. ${ }^{15}$ In this study, an experimental immature bovine tooth model was designed to investigate the effect of filling the cervical and middle regions of root canals with ProRoot MTA, CEM Cement, and Biodentine on the fracture resistance of immature teeth. The null hypothesis was that filling the cervical and middle regions of root canals using biosilicate cements would not increase the fracture resistance of immature teeth.

\section{Materials and Methods}

Seventy freshly extracted bovine incisors were collected and disinfected in $2.5 \%$ sodium hypochlorite solution for 30 minutes. The crowns and roots were sectioned $8 \mathrm{~mm}$ above and $12 \mathrm{~mm}$ below the cementoenamel junction (CEJ), respectively, resulting in a standard length of $20 \mathrm{~mm}$. The dentinal thickness of the most cervical part of the roots just below the CEJ was measured using a digital caliper. Those with $8 \pm 0.5 \mathrm{~mm}$ buccolingual and $6 \pm 0.5 \mathrm{~mm}$ mesiodistal dimensions were selected in an attempt to control the confounding factor of root dimension. The internal canal anatomy of each specimen was then examined using two radiographic images taken from the buccolingual and mesiodistal direction. Teeth with cracks and/or fractures were excluded. To standardize the specimens, the entire length of each root canal was then enlarged in a coronoapical direction using a 2.2-mm diameter water-cooled fissure bur (Jota AG, Rüthi, Switzerland). During the procedure, the specimens were maintained in moist gauze to prevent dehydration. Two more digital radiographic images were then taken from mesiodistal and buccolingual directions, and the mean dentine thickness of the most coronal portion of the roots was measured to an accuracy of $0.01 \mathrm{~mm}$. The specimens were then allocated into five groups according to the coronal dentine thickness. Group I: Biodentine. The liquid was added to the powder within the capsule and mechanically mixed for 30 seconds using an amalgamator (Linker, Zhengzhou, Henan, China). The slurry was then applied into each canal using an amalgam carrier (ASA Dental, Massarosa [Lu], Italy) and then adapted to root canal walls using a plugger (Dentsply Sirona Endodontics, Ballaigues, Switzerland) at minimal pressure. The root-filling material was built up to the level of the CEJ on the labial area of the crown ( - Fig. 1B). Excess cement was removed from the access cavity with a wet cotton pellet. Group II: The cervical and middle regions of the root canal were filled with tooth-colored ProRoot MTA (Dentsply Tulsa Dental, Tulsa, Oklahoma, United States). One gram of MTA powder was placed in a clean amalgam capsule and mixed with $0.33 \mathrm{~mL}$ distilled water as described by Nekoofar et al ${ }^{16}$ and adapted to the canal with a plugger (Dentsply International) using minimal pressure as in Group I. Group III: To standardize the mixing technique, $1 \mathrm{~g}$ CEM Cement powder (BioniqueDent) and $0.33 \mathrm{~mL}$ liquid were added to an empty amalgam capsule, mixed mechanically using an amalgamator at $4000 \mathrm{rpm}$ for 30 seconds and placed into the canal using minimal pressure as above. Group IV: Each canal was filled with gutta-percha points (Gapadent, Tianjin, China) and AH26 sealer (Dentsply De-Trey GmbH, Konstanz, Germany) using a vertical condensation technique: Size 80 gutta-percha points were cut into 3 to $4 \mathrm{~mm}$ sections, heated and placed into the canal, and compacted with a plugger up to the CEJ. After placement of the root fillings, a digital radiographic image was taken of all specimens in the buccolingual direction to assess the quality of the filling. Group V: The root canal was filled with dry cotton wool. During the canal filling procedures, the specimens were kept in moist gauze to prevent dehydration. The access cavities of all specimens were then filled with Coltosol and the specimens were stored at $37^{\circ} \mathrm{C}$ in a fully saturated atmosphere for 24 hours. To mount the specimens, a metal cylindrical mold $(25-\mathrm{mm}$ diameter $\times$
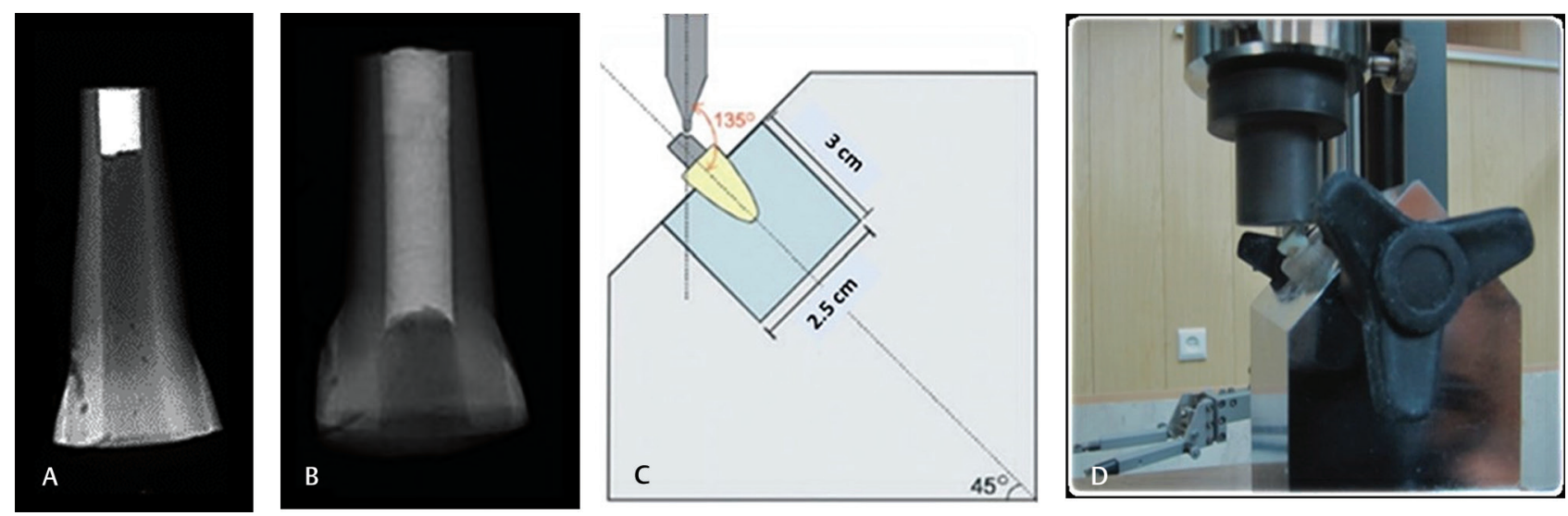

Fig. 1 (A) Radiographic view of the Coltosol apical plug, (B) obturation of the entire root canal with Biodentine, (C) schematic view of the jig of the universal testing machine, and (D) relation of the indenter and the sample 
30-mm high) was designed. The mold was filled with selfcure acrylic resin (Acropras, Eshtehard, Iran) and specimens were then immersed up to $2 \mathrm{~mm}$ below the CEJ of the buccal surface to simulate the biological width. To simulate traumatic forces on the middle third of the tooth crowns, a metal jig was designed as described by Melo et al $^{17}$ ( - Fig. 1D) to produce compressive loads at a crosshead speed of $5 \mathrm{~mm}$ $\mathrm{min}^{-1}$ in a universal testing machine (Zwick/Roell Z050, Ulm, Germany) until fracture. The apparatus fixed the specimens at a $45^{\circ}$ angle in such a way that the load was applied by the indenter at $135^{\circ}$ to the long axis of the tooth from the buccal direction (-Fig. 1C). The maximum load required to fracture the specimens was recorded in Newton (N). To standardize the length of the apical filling, 15-mm customized wooden cylinders were fitted into the root canals through the coronal access, and the apical region of all specimens was filled with a noneugenol temporary filling material, Coltosol (Coltene, Altstätten, Switzerland), and a digital radiograph exposed (-Fig. 1A). The wooden cylinder was removed after 24-hour incubation at $37^{\circ} \mathrm{C}$ in a fully saturated humidity. Then, the remaining root canal system was filled using one of the test materials producing five groups each of 14 specimens.

\section{Statistical Analysis}

Fracture resistance values were calculated as means and standard deviations (SDs). To compare the intergroup differences, one-way variance analysis, and for intergroup comparison (two by two comparison), Tukey's test at a 5\% level of significance was used. Mode of fracture was evaluated and analyzed by chi-squared tests and corrected by the Fisher's exact test. Mode of fracture was evaluated and five types of fracture were seen: (1) enamel fracture, (2) crown fracture, (3) coronal third fracture, (4) mid-third fracture, and (5) apical third fracture (-Table 1 ).

\section{Results}

The mean fracture strength values and SD of the groups are shown in - Table 1. Biodentine and ProRoot MTA groups had the greatest fracture resistance. Significant differences were found between Biodentine and ProRoot MTA groups compared with the control $(p<0.005)$. No significant difference was seen between CEM Cement and the gutta-percha groups compared with the control group. Mode of fracture and the distribution in each group are shown in - Table 1. The distribution of fractures was not significantly different between the five groups.

\section{Discussion}

Resistance to fracture for root-filled teeth depends to a large degree on the amount of remaining tooth structure. ${ }^{18}$ Due to thin dentinal walls and susceptibility to fracture, particularly in the cervical area, ${ }^{19,20}$ the survival of endodontically treated immature teeth is of concern. The apical third of immature teeth may not bear significant masticatory forces; therefore, the remaining dentine in the coronal and middle portion of the root provides the main resistance against loading forces. ${ }^{21}$ The present study was designed to investigate the ability of several calcium silicate-based cements (ProRoot MTA, CEM Cement, and Biodentine ${ }^{\mathrm{TM}}$ ) to reinforce immature teeth when placed in the coronal and middle third of the root canal.

A bovine root model was developed to simulate the clinical condition of immature teeth, that is, Cvek's Stage 3 root development model with two-thirds of final length ${ }^{5}$ and a root-to-canal ratio of $\sim 1: 1$ at the CEJ in the mesiodistal direction. In an attempt to standardize the samples and reduce confounding variables, the apical third of all specimens was filled with a noneugenol temporary filling material. To eliminate the effect of a calcium hydroxide dressing, none was placed in the root canals so that the specific effect of calcium silicate-based cements could be evaluated.

Despite the differences between human and bovine teeth, Sano et $\mathrm{al}^{22}$ have demonstrated similarities in ultimate tensile strength, dentine modulus of elasticity, and number and distribution of dentinal tubules between bovine and human teeth. In addition, few anatomical and morphological variations have been found in bovine incisors in comparison with human teeth. ${ }^{23-26}$ Therefore, this model provides a consistent methodology to assess new treatment modalities for immature teeth. Similar to previous studies, ${ }^{27-29}$ an experimental immature tooth model using bovine teeth was thus employed. To improve the drawbacks of MTA, such as difficult handling properties and prolonged setting time, a variety of silicate-based cements such as CEM Cement and Biodentine have been introduced. ${ }^{30,31}$ It has been claimed that Biodentine sets in 10 minutes and can be used as a single application bulk restorative material without cavity conditioning, ${ }^{32}$ in

Table 1 Mean value and standard deviation of fracture strength and mode of fracture of the samples

\begin{tabular}{|c|c|c|c|c|c|c|}
\hline \multirow[t]{2}{*}{ Experimental group } & \multirow{2}{*}{$\begin{array}{l}\text { Fracture strength } \\
\text { values, mean + SD }\end{array}$} & \multicolumn{5}{|c|}{ Mode of fracture of the samples (\%) } \\
\hline & & Crown Fx. & VRF & $\begin{array}{l}\text { One-third } \\
\text { middle root } \\
\text { Fx. }\end{array}$ & $\begin{array}{l}\text { One-third } \\
\text { cervical root } \\
\text { Fx. }\end{array}$ & Enamel Fx. \\
\hline Control & $1451.6 \pm 332.1$ & 0.0 & 28.6 & 50 & 14.3 & 7.1 \\
\hline Gutta-percha & $1873.5 \pm 579.8$ & 7.1 & 14.3 & 14.3 & 64.3 & 0.0 \\
\hline CEM & $1860.8 \pm 370.6$ & 14.3 & 21.4 & 14.3 & 50.0 & 0.0 \\
\hline MTA & $2103.6 \pm 549.8$ & 0.0 & 21.4 & 14.3 & 64.3 & 0.0 \\
\hline Biodentine & $2196.1 \pm 575.6$ & 0.0 & 35.7 & 28.6 & 21.4 & 14.3 \\
\hline
\end{tabular}

Abbreviations: CEM, calcium-enriched mixture; Fx., fracture; MTA, mineral trioxide aggregate; SD, standard deviation; VRF, vertical root fracture. 
other words, it acts as a dentine substitute material, a temporary filling material and establishes an immediate seal against oral fluids. ${ }^{33}$ Therefore, in the management of immature teeth with minimum root dentine, it can be an appropriate choice. According to the results of this study, Biodentine specimens had significantly higher fracture resistance values compared with the control group. This finding is in agreement with the results of the study by Cauwels et al. ${ }^{4}$ In accordance with Bortoluzzi et al, ${ }^{27}$ ProRoot MTA specimens also had significantly higher fracture resistance values than the control group. In the present study, no significant difference was found between the fracture strength values of Biodentine and ProRoot MTA groups. However, no significant difference was found in fracture resistance values between CEM Cement, gutta-percha and sealer, and the control group. Considering the fact that ProRoot MTA, ${ }^{10,34,35}$ CEM Cement, ${ }^{36}$ and Biodentine ${ }^{10,37}$ are bioactive and are associated with apatite formation after exposure to body fluids containing phosphorus, they may provide these higher values of fracture resistance due to apatite crystalline formation along the cement and dentine interface and within interfacial dentine. Indeed, the fracture resistance values of specimens filled with calcium silicate-based cements may increase over time. Longer incubation time and exposure of the specimens to simulated body fluid are suggested for the future studies.

\section{Conclusion}

Under the limitations of the present study, it was concluded that root filling with ProRoot MTA and Biodentine increased the fracture resistance of the simulated immature teeth compared with control specimens with no root filling. No significant difference was seen between CEM Cement, gutta-percha/sealer, and controls.

\section{Funding}

This study was supported by a grant from Tehran University of Medical Sciences (grant no. 14667).

\section{Conflict of Interest}

None declared.

\section{References}

1 Bakhtiar H, Esmaeili S, Fakhr Tabatabayi S, Ellini MR, Nekoofar $\mathrm{MH}$, Dummer PM. Second-generation platelet concentrate (Platelet-rich fibrin) as a scaffold in regenerative endodontics: a case series. J Endod 2017;43(3):401-408

2 Frank AL. Therapy for the divergent pulpless tooth by continued apical formation. J Am Dent Assoc 1966;72(1):87-93

3 Andreasen JO, Farik B, Munksgaard EC. Long-term calcium hydroxide as a root canal dressing may increase risk of root fracture. Dent Traumatol 2002;18(3):134-137

4 Cauwels RG, Pieters IY, Martens LC, Verbeeck RM. Fracture resistance and reinforcement of immature roots with gutta percha, mineral trioxide aggregate and calcium phosphate bone cement: a standardized in vitro model. Dent Traumatol 2010;26(2):137-142

5 Cvek M. Prognosis of luxated non-vital maxillary incisors treated with calcium hydroxide and filled with gutta-percha.
A retrospective clinical study. Endod Dent Traumatol 1992;8(2):45-55

6 Pace R, Giuliani V, Pini Prato L, Baccetti T, Pagavino G. Apical plug technique using mineral trioxide aggregate: results from a case series. Int Endod J 2007;40(6):478-484

7 Nosrat A, Asgary S, Eghbal MJ, Ghoddusi J, Bayat-Movahed S. Calcium-enriched mixture cement as artificial apical barrier: a case series. J Conserv Dent 2011;14(4):427-431

8 Torabinejad M, Parirokh M, Dummer PMH. Mineral trioxide aggregate and other bioactive endodontic cements: an updated overview - part II: other clinical applications and complications. Int Endod J 2018;51(3):284-317

9 Asgary S, Nosrat A, Seifi A. Management of inflammatory external root resorption by using calcium-enriched mixture cement: a case report. J Endod 2011;37(3):411-413

10 Prati C, Gandolfi MG. Calcium silicate bioactive cements: biological perspectives and clinical applications. Dent Mater 2015;31(4):351-370

11 Karapinar-Kazandag M, Basrani B, Tom-Kun Yamagishi V, Azarpazhooh A, Friedman S. Fracture resistance of simulated immature tooth roots reinforced with MTA or restorative materials. Dent Traumatol 2016;32(2):146-152

12 Porter ML, Bertó A, Primus CM, Watanabe I. Physical and chemical properties of new-generation endodontic materials. J Endod 2010;36(3):524-528

13 Akbari M, Zebarjad SM, Nategh B, Rouhani A. Effect of nano silica on setting time and physical properties of mineral trioxide aggregate. J Endod 2013;39(11):1448-1451

14 Noh YS, Chung SH, Bae KS, et al. Mechanical properties and microstructure analysis of mineral trioxide aggregate mixed with hydrophilic synthetic polymer. J Biomed Mater Res B Appl Biomater 2015;103(4):777-782

15 Asgary S, Eghbal MJ, Parirokh M. Sealing ability of a novel endodontic cement as a root-end filling material. J Biomed Mater Res A 2008;87(3):706-709

16 Nekoofar MH, Aseeley Z, Dummer PM. The effect of various mixing techniques on the surface microhardness of mineral trioxide aggregate. Int Endod J 2010;43(4):312-320

17 Melo MP, Valle AL, Pereira JR, Bonachela WC, Pegoraro LF, Bonfante G. Evaluation of fracture resistance of endodontically treated teeth restored with prefabricated posts and composites with varying quantities of remaining coronal tooth structure. J Appl Oral Sci 2005;13(2):141-146

18 Marchi GM, Mitsui FH, Cavalcanti AN. Effect of remaining dentine structure and thermal-mechanical aging on the fracture resistance of bovine roots with different post and core systems. Int Endod J 2008;41(11):969-976

19 Goldberg F, Kaplan A, Roitman M, Manfré S, Picca M. Reinforcing effect of a resin glass ionomer in the restoration of immature roots in vitro. Dent Traumatol 2002;18(2):70-72

20 Katebzadeh N, Dalton BC, Trope M. Strengthening immature teeth during and after apexification. J Endod 1998;24(4):256-259

21 Talati A, Disfani R, Afshar A, Fallah Rastegar A. Finite element evaluation of stress distribution in mature and immature teeth. Iran Endod J 2007;2(2):47-53

22 Sano H, Ciucchi B, Matthews WG, Pashley DH. Tensile properties of mineralized and demineralized human and bovine dentin. J Dent Res 1994;73(6):1205-1211

23 Nakamichi I, Iwaku M, Fusayama T. Bovine teeth as possible substitutes in the adhesion test. J Dent Res 1983;62(10):1076-1081

24 Ruse ND, Smith DC. Adhesion to bovine dentin--surface characterization. J Dent Res 1991;70(6):1002-1008

25 Saunders WP. The shear impact retentive strengths of four dentine bonding agents to human and bovine dentine. J Dent $1988 ; 16(5): 233-238$ 
26 Schilke R, Lisson JA, Bauss O, Geurtsen W. Comparison of the number and diameter of dentinal tubules in human and bovine dentine by scanning electron microscopic investigation. Arch Oral Biol 2000;45(5):355-361

27 Bortoluzzi EA, Souza EM, Reis JM, Esberard RM, Tanomaru-Filho M. Fracture strength of bovine incisors after intra-radicular treatment with MTA in an experimental immature tooth model. Int Endod J 2007;40(9):684-691

28 Carvalho CA, Valera MC, Oliveira LD, Camargo CH. Structural resistance in immature teeth using root reinforcements in vitro. Dent Traumatol 2005;21(3):155-159

29 Schmoldt SJ, Kirkpatrick TC, Rutledge RE, Yaccino JM. Reinforcement of simulated immature roots restored with composite resin, mineral trioxide aggregate, gutta-percha, or a fiber post after thermocycling. J Endod 2011;37(10):1390-1393

30 Bolhari B, Nekoofar MH, Sharifian M, Ghabrai S, Meraji N, Dummer PM. Acid and microhardness of mineral trioxide aggregate and mineral trioxide aggregate-like materials. J Endod 2014;40(3):432-435

31 Kadić S, Baraba A, Miletić I, et al. Push-out bond strength of three different calcium silicate-based root-end filling materials after ultrasonic retrograde cavity preparation. Clin Oral Investig 2018;22(3):1559-1565

32 Kaur M, Singh H, Dhillon JS, Batra M, Saini M. MTA versus Biodentine: review of literature with a comparative analysis. J Clin Diagn Res 2017;11(8):ZG01-ZG05

33 Laurent P, Camps J, De Méo M, Déjou J, About I. Induction of specific cell responses to a $\mathrm{Ca}(3) \mathrm{SiO}(5)$-based posterior restorative material. Dent Mater 2008;24(11):1486-1494

34 Reyes-Carmona JF, Felippe MS, Felippe WT. Biomineralization ability and interaction of mineral trioxide aggregate and white Portland cement with dentin in a phosphate-containing fluid. J Endod 2009;35(5):731-736

35 Sarkar NK, Caicedo R, Ritwik P, Moiseyeva R, Kawashima I. Physicochemical basis of the biologic properties of mineral trioxide aggregate. J Endod 2005;31(2):97-100

36 Asgary S, Eghbal MJ, Parirokh M, Ghoddusi J. Effect of two storage solutions on surface topography of two root-end fillings. Aust Endod J 2009;35(3):147-152

37 Kim JR, Nosrat A, Fouad AF. Interfacial characteristics of Biodentine and MTA with dentine in simulated body fluid. J Dent 2015;43(2):241-247 